\title{
Discrete unified gas kinetic scheme for all Knudsen number flows. IV. Strongly inhomogeneous fluids
}

\author{
Baochao Shan ${ }^{1}$, Peng Wang ${ }^{1}$, Yonghao Zhang ${ }^{2}$, Zhaoli Guo ${ }^{1 *}$ \\ 1. State Key Laboratory of Coal Combustion, Huazhong University of Science and Technology, Wuhan, Hubei 430000, China \\ 2. James Weir Fluids Laboratory, Department of Mechanical and Aerospace Engineering, University of Strathclyde, Glasgow G1 1 XJ, UK \\ * Corresponding e-mail address: $\underline{\text { lguo@hust.edu.cn }}$
}

Abstract: This work is an extension of the discrete unified gas kinetic scheme (DUGKS) from rarefied gas dynamics to strongly inhomogeneous dense fluid systems. The fluid molecular size can be ignored for dilute gases, while the nonlocal intermolecular collisions and the competition of solid-fluid and fluid-fluid interactions play an important role for surface-confined fluid flows at the nanometer scale. The non-equilibrium state induces strong fluid structural inhomogeneity and anomalous fluid flow dynamics. According to the previous kinetic model [Z. L. Guo et al., Phy. Rev. E 71, 035301 (2005)], the long-range intermolecular attraction is modeled by the mean-field approximation, and the volume exclusion effect is considered by hard-sphere potential in the collision operator. The kinetic model is solved by the DUGKS, which has the characteristics of asymptotic preserving, low dissipation, second order accuracy and multidimensional nature. Both static fluid structure and dynamic flow behaviors are calculated and validated with Monte Carlo or molecular dynamics results. It is shown that the flow of dense fluid systems tends to that of rarefied gases as the dense degree decreases or the mean flow path increases. The DUGKS is proved to be applicable to simulate such non-equilibrium dense fluid systems.

Key words: discrete unified gas kinetic scheme; dense gas; nanometer fluid flow; non-equilibrium effects 


\section{Introduction}

The hydrodynamics of nanoscale fluid systems exhibit many peculiar behaviors comparing to that at the macroscopic level [1], which has drawn growing interests in the research of lab-on-a-chip [2], storage, conversion and exploitation of energy [3-6], water purification $[7,8]$, nano-manufacturing $[9,10]$, carbon sequestration in Metal Organic Frameworks [11], gas separation [12,13] and so on. Although the particle-based molecular dynamics (MD) and direct simulation of Monte Carlo (DSMC) have been commonly used to study such systems, these techniques are usually computationally intensive [1], and suffer huge statistical noises, especially for flows near the equilibrium state [14] or in the high density regime [15]. Therefore, a numerical scheme with high accuracy and applicability to a wide range of flow regimes is desirable for the study of nanometer scale fluid flows.

The Knudsen number $(K n)$, which is defined as the ratio of fluid molecular mean free path (MFP) to the characteristic length of flow field [14], is normally taken as the criterion number to characterize flow regimes from the continuum flow $(K n<0.001)$ to the free molecular flow $(K n>10)$ in rarefied gas dynamics. The Navier-Stokes (NS) equation can be adopted to simulate fluid flow in the continuum flow regime, while the NS equation with slip boundary condition is usually employed in the slip flow regime $(0.001<K n<0.1)$, where the rarefaction effects can no longer be neglected [5,16-18]. However, the NS equation (with slip boundary condition) fails to capture the non-equilibrium effects in more rarefied flow regimes, e.g., the transition and free molecular flow regimes, where the continuum assumption becomes totally invalid [15]. Besides, it also fails when fluid properties or transport coefficients vary significantly over a molecular size [19]. Consequently, the NS equation cannot be employed to 
capture nanoscale fluid behavior, since both the cases may happen in a denes fluid system at the nanometer scale.

The Boltzmann equation is well-recognized to work in all the flow regimes ranging from continuum flow to free molecular flow $[20,21]$. However, it is only valid for dilute gases with homogeneous properties, i.e., satisfying the following conditions: (1) ignorable molecular size; (2) localized binary collision between molecules; (3) molecular chaos hypothesis [1,15,22]. For a dense fluid system at the nanometer scale, molecular size cannot be ignored since it is comparable to the characteristic length or the MFP. Thus, the Boltzmann equation breaks down for such systems.

The Boltzmann equation was extended into dense gas system by Enskog [23] and later modified by van Beijeren and Ernst [24], known as the Enskog theory and revised Enskog theory, respectively. Although molecular size and the collisional transfer of momentum and energy (non-local collisions) are considered in such theory, it still assumes molecular chaos and uses the rigid spherical model [23]. In addition, molecular interactions (fluid-fluid and fluid-solid) become predominant on dynamical and structural properties of dense fluids in the nanoscale fluid flows. Therefore, the Enskog theory is also not sufficient to describe the state of dense fluids system at the nanometer scale. To overcome this limitation, the effects of a long-range smooth attractive tail is added to the hard-core repulsion of the Enskog equation, known as the Enskog-Vlasov equation [25-27], to model the intermolecular potential effects in dense fluids, where the long-range interactions are dealt with by a collective meanfield.

Based on the Enskog-Vlasov equation, the nanoscale fluid flow is studied by Davis [28] and Vanderlick [29,30], where the kinetic equation yields the exact Yvon-Born-Green 
equations for the density distributions at equilibrium. The molecular size, non-local collisions and molecular interactions (fluid-solid and fluid-fluid) were simultaneously considered. However, the collision operator in their theory is quite difficult in practical applications. Later, a tractable kinetic model was proposed by Guo et al. [22] to account for the strong inhomogeneity in dense fluid systems. Following the Chapman-Enskog analysis, the original kinetic equation was employed to study the equilibrium and dynamic behaviors of confined fluids on the macroscale level. However, only the no-slip cases were studied in their paper. Besides, the hydrodynamic equation [22] is only applicable to continuum flows, since it retains up to the first order terms in the Chapman-Enskog series. Thus, the non-equilibrium effects are not properly captured. In this study, the discrete unified gas-kinetic scheme (DUGKS) [31] is extended to solve the kinetic model [22] for strongly inhomogeneous confined fluid systems, which can capture the rarefaction effects as well.

Combining the advantages of the lattice Boltzmann method (LBM) [32] and the unified gas kinetic scheme (UGKS) [15], the DUGKS [31] was proposed recently for rarefied gas flows, which is applicable to the entire flow regimes. It has been successfully applied to low-speed isothermal flows ranging from the continuum to free molecular flow regimes [31], compressible flows considering heat transfer and shock discontinuity [33], flows of binary gas mixtures [14], Boussinesq flows [34], multiscale heat transfer [35-37], thermally induced non-equilibrium flows [38], rarefied gas flow in micro-channels [39], solid-liquid phase change problems [40], immiscible two phase flows [41] etc.. The capability of the DUGKS to tackle multiscale problems has been thoroughly discussed in these studies, and a rigorous theoretical analysis of its unified preserving properties was also made recently [42]. However, the DUGKS is based on 
the Boltzmann equation, which is not sufficient for dense fluid systems with strong inhomogeneity at the nanometer scale $[1,4,9,22]$.

The purpose of this paper is to extend the DUGKS to non-equilibrium dense fluid systems with strong inhomogeneity at the nanometer scale based on the kinetic model [22], where the effects of volume exclusion and long-range fluid-fluid and fluid-wall interactions are simultaneously taken into account.

\section{Kinetic model for nanoscale dense fluid system}

The Enskog theory considers the effects of finite size of molecules and non-local collisions of hard-sphere fluids $[4,23]$, which are ignored in the Boltzmann equation. Combining the Enskog equation and the mean-field theory to account for the volume exclusion effects and the long-range intermolecular attractions, respectively, the evolution of velocity distribution function for a dense fluid can be described by the following kinetic equation [22]

$$
\partial_{t} f+\boldsymbol{\xi} \cdot \nabla_{r} f-m^{-1} \nabla_{r}\left(\phi_{e x t}+\phi_{m}\right) \cdot \nabla_{\xi} f=\Omega(f)
$$

where $f(\boldsymbol{r}, \boldsymbol{\xi}, t)$ is the velocity distribution function of molecular velocity $\boldsymbol{\xi}$ at spatial position $\boldsymbol{r}$ and time $t, \partial_{t}$ represents partial derivative in terms of time $t, m$ is the molecular mass, $\nabla_{r}$ and $\nabla_{\boldsymbol{\xi}}$ represent gradient operators in terms of space $\boldsymbol{r}$ and velocity $\boldsymbol{\xi}$, respectively; $\phi_{\text {ext }}$ is the external potential term, $\phi_{m}$ relates to the attractive part of the fluid-fluid potential, and $\Omega(f)$ is the extended Enskog collision operator. Following the projection method for hard-sphere fluids $[9,43], \Omega(f)$ can be further divided as the superposition of a Boltzmann collision term $\Omega_{B}$ and an excess collision term $\Omega_{E}$. The Boltzmann part $\Omega_{B}$ is modeled by the Bhatnagar-Gross-Krook relaxation process [22], 


$$
\Omega_{B}=-\frac{f-f^{e q}}{\tau},
$$

where $\tau$ is the relaxation time, and $f^{e q}$ is the Maxwellian local equilibrium distribution function

$$
f^{e q}=n\left(\frac{1}{2 \pi R T}\right)^{3 / 2} \exp \left[-\frac{(\boldsymbol{\xi}-\boldsymbol{u})^{2}}{2 R T}\right]
$$

where $n$ is the number density, $R$ is the gas constant, $T$ is the constant temperature of the isothermal system, $\boldsymbol{u}$ is the flow velocity. The macro flow variables can be calculated from the moments of the distribution function,

$$
n=\int f d \boldsymbol{\xi}, \boldsymbol{u}=n^{-1} \int \boldsymbol{\xi} f d \boldsymbol{\xi}
$$

Note that only the isothermal case is considered in this paper, and the temperature is given as a constant. The excess collision term $\Omega_{E}$ accounting for volume exclusion effects of intermolecular repulsion is expressed as [22]

$$
\Omega_{E}=-V_{0} f^{e q}(\boldsymbol{\xi}-\boldsymbol{u}) \cdot[2 \boldsymbol{A} \chi(\bar{n})+\boldsymbol{B} \bar{n}]
$$

where $V_{0}$ is related to molecular diameter $\sigma$, i.e., $\quad V_{0}=2 \pi \sigma{ }^{3} / 3 \quad[23,44]$; $\bar{n}=\int w\left(\boldsymbol{r}^{\prime}\right) n\left(\boldsymbol{r}+\boldsymbol{r}^{\prime}\right) d \boldsymbol{r}^{\prime}$ is the local average density (LAD) with $w(\boldsymbol{r})$ being a weight function [45], which was commonly used in the free energy density functional theory (DFT) to study inhomogeneous fluid systems $[29,45] ; \chi$ is the radial distribution function (RDF) for homogenous hard-sphere fluids [46]. To account for the inhomogeneity of dense fluid system, the RDF $\chi$ in Eq.(5) is evaluated with the LAD, rather than the local density $n$. Meanwhile, the parameters $\boldsymbol{A}$ and $\boldsymbol{B}$ are two gradient operators defined by [22]

$$
\boldsymbol{A}=\frac{1}{D} \int_{\left|\boldsymbol{r}^{\prime}\right|<\sigma / 2} \boldsymbol{r}^{\prime} \bar{n}\left(\boldsymbol{r}+\boldsymbol{r}^{\prime}\right) d \boldsymbol{r}^{\prime}
$$

and 


$$
\boldsymbol{B}=\frac{1}{D} \int_{\left|\boldsymbol{r}^{\prime}\right|<\sigma / 2} \boldsymbol{r}^{\prime} \chi\left[\bar{n}\left(\boldsymbol{r}+\boldsymbol{r}^{\prime}\right)\right] d \boldsymbol{r}^{\prime}
$$

where $\sigma$ is effective molecular diameter, and $D$ is equal to $\pi \sigma^{5} / 120$.

The external potential $\phi_{e x t}$ includes all external potentials, such as the wall potential $\phi_{w}$ and that driving the fluid to flow. The wall potential can be represented by the $10-4-3$ potential for a planar wall [29]

$$
\phi_{w}(z)=2 \pi \varepsilon_{w f}\left[\frac{2}{5}\left(\frac{\sigma_{w f}}{z}\right)^{10}-\left(\frac{\sigma_{w f}}{z}\right)^{4}-\frac{\sigma_{w f}^{4}}{3 \Delta(z+0.61 \Delta)^{3}}\right], \Delta=\sigma_{w f} / \sqrt{2}
$$

or the $10-4$ potential [47]

$$
\phi_{w}(z)=2 \pi \varepsilon_{w f}\left[\frac{4}{5}\left(\frac{\sigma_{w f}}{z}\right)^{10}-\left(\frac{\sigma_{w f}}{z}\right)^{4}\right]
$$

where $\varepsilon_{w f}$ and $\sigma_{w f}$ are the energy and range parameters of wall-fluid interactions, respectively; and $z$ is the perpendicular distance from the wall. Note that the $10-4-3$ wall potential is a result of the integration of a continuous distribution of all the solid molecules interacting with gas molecules through the $12-6$ L-J potential. Consequently, the $10-4-3$ wall potential is approximately equivalent to the $12-6$ potential of fluid-solid molecules in the MD simulations. For more general geometrics, the potential at a position can be measured from those of all solid molecules. The empirical parameters between the interactive molecules are chosen exactly the same as those in the MD simulations.

The mean-field theory is adopted to account for the long-range intermolecular attraction, where a gas molecular is considered to move under the average attraction of molecules in the system [48]. According to the decomposition principle of the pair-wise intermolecular potential [49], the molecular interaction part acts when the distance between molecules is larger than 
effective diameter $\sigma$, and thus, $\phi_{m}$ in Eq.(1) can be expressed as

$$
\phi_{m}(\boldsymbol{r})=\int_{\left|r^{\prime}\right|>\sigma} n\left(\boldsymbol{r}+\boldsymbol{r}^{\prime}\right) \phi_{a t t}\left(\left|\boldsymbol{r}^{\prime}\right|\right) d \boldsymbol{r}^{\prime}
$$

where $\phi_{\text {att }}$ is the attractive part of the Lennard-Jones (LJ) fluids, which can be represented as

$$
\phi_{a t t}(r)=4 \varepsilon_{f f}\left[\left(\frac{\sigma_{f f}}{r}\right)^{12}-\left(\frac{\sigma_{f f}}{r}\right)^{6}\right],
$$

where $\varepsilon_{f f}$ and $\sigma_{f f}$ are the energy and range parameters of fluid-fluid interactions, respectively; and $r$ is the distance between two fluid molecules. Meanwhile, the interaction range of wall atoms and fluid atoms can be represented by the effective diameter $\sigma$ as $[9,50]$

$$
\sigma \approx \frac{1+a_{1} T_{r}}{1+a_{2} T_{r}+a_{3} T_{r}^{2}}
$$

where $T_{r}=k_{B} T / \varepsilon$ is the reduced temperature, with $a_{1}=0.2977, a_{2}=0.33163$ and $a_{3}=$ 0.00104771.

\section{DUGKS for the kinetic model}

In this section, the DUGKS will be employed to solve the kinetic equation (1). Before implementation, a transformation is conducted on Eq.(1) for convenience

$$
\partial_{t} f+\boldsymbol{\xi} \cdot \nabla_{r} f=\Omega_{B}+\boldsymbol{G}
$$

where $\boldsymbol{G}$ is a total force term as a combination of volume exclusion effects, long-range intermolecular attraction, surrounding wall potentials and other outside forces, which in the current work can be expressed as

$$
\boldsymbol{G}=-\left[\nabla \phi_{e x t}+\nabla \phi_{m}+V_{0} R T(2 \boldsymbol{A} \chi(\bar{n})+\boldsymbol{B} \bar{n})\right] \frac{\boldsymbol{\xi}-\boldsymbol{u}}{R T} f^{e q}
$$

where the derivative of distribution function $f$ in terms of particle velocity $\nabla_{\xi} f$ was approximated by its equilibrium state $\nabla_{\xi} f^{e q}$, due to the fact that $f^{e q}$ is the leading part of the 
distribution $f$ and the gradient of $f^{e q}$ has the most important contribution to the gradient of $f$ [48], especially for high fluid density cases as in the current work

$$
\nabla_{\xi} f \approx \nabla_{\xi} f^{e q}=-\frac{\boldsymbol{\xi}-\boldsymbol{u}}{R T} f^{e q}
$$

Since the original DUGKS does not include the external force term $\boldsymbol{G}$, we will update the Eq.(13) by two steps: (1) employ the standard updating rules as originally described in $[14,31,33]$, see sections 2.1 and $2.2 ;(2)$ treat the external force term $\boldsymbol{G}$ by the Strang splitting technique [51-53], see section 2.3.

\subsection{Updating in the standard DUGKS}

For the original DUGKS without considering the external force term $\boldsymbol{G}$, Eq.(13) can be written as

$$
\partial_{t} f+\boldsymbol{\xi} \cdot \nabla_{r} f=\Omega_{B}
$$

Adopting the midpoint rule for time integration of the convection term, and the trapezoidal rule for the collision term of evolution equation Eq.(16), we can discretize it into the following form for cell $j\left(\boldsymbol{r}_{j}\right.$ is the cell center) from time $t_{n}$ to $t_{n+1}$ as

$$
f_{j}^{n+1}-f_{j}^{n}+\frac{\Delta t}{\left|V_{j}\right|} F^{n+1 / 2}=\frac{\Delta t}{2}\left(\Omega_{j}^{n+1}-\Omega_{j}^{n}\right),
$$

where the superscript $(n+1)$ and $n$ represent time $t_{n+1}$ and $t_{n}$, respectively; the subscript $j$ represents space $\boldsymbol{r}_{\mathrm{j}}$ at the cell center, $F^{n+1 / 2}$ is micro flux across the cell interface, i.e.,

$$
F^{n+1 / 2}=\int_{\partial V_{j}}(\boldsymbol{\xi} \cdot \boldsymbol{n}) f\left(\boldsymbol{r}, t_{n+1 / 2}\right) d \boldsymbol{S}
$$

where $\partial V_{j}$ and $\left|V_{j}\right|$ are cell surface and cell volume of the cell $V_{j}$, respectively; $\boldsymbol{n}$ is the outward unit vector normal to the surface.

By introducing two auxiliary distribution functions $\tilde{f}$ and $\tilde{f}^{+}$as 


$$
\begin{gathered}
\tilde{f}=f-\frac{\Delta t}{2} \Omega_{B}=\frac{2 \tau+\Delta t}{2 \tau} f-\frac{\Delta t}{2 \tau} f^{e q}, \\
\tilde{f}^{+}=f+\frac{\Delta t}{2} \Omega_{B}=\frac{2 \tau-\Delta t}{2 \tau+\Delta t} \tilde{f}+\frac{2 \Delta t}{2 \tau+\Delta t} f^{e q},
\end{gathered}
$$

the Eq.(16) can be rewritten as

$$
\tilde{f}_{j}^{n+1}=\tilde{f}_{j}^{+, n}-\frac{\Delta t}{\left|V_{j}\right|} F^{n+1 / 2} .
$$

Since the BGK collision operator $\Omega_{B}$ satisfies the following conservative laws

$$
\int \Omega_{B} d \boldsymbol{\xi}=0, \quad \int \boldsymbol{\xi} \Omega_{B} d \boldsymbol{\xi}=0
$$

the evolution can be done explicitly according to Eq.(21) by tracking auxiliary distribution function $\tilde{f}$, instead of the original distribution function $f$. The density and velocity can be calculated as

$$
n=\int \tilde{f} d \boldsymbol{\xi}, n \boldsymbol{u}=\int \boldsymbol{\xi} \tilde{f} d \boldsymbol{\xi}
$$

\subsection{Flux evaluation}

In order to update $\tilde{f}$ from $t_{n}$ to $t_{n+1}$ according to Eq.(21), the micro flux across the cell interface $\partial V_{j}$ needs to be evaluated first, the key point of which is to reconstruct the original distribution function $f^{n+1 / 2}$ at time $t_{n+1 / 2}$ on cell interface. Similar to the treatment in the above updating rule, we integrate Eq.(16) along the characteristic line within a half time step, i.e., $h$ $=\Delta t / 2$ with the trapezoidal rule for the collision term

$$
f\left(\boldsymbol{r}_{i j}, \boldsymbol{\xi}, t_{n}+h\right)-f\left(\boldsymbol{r}_{i j}-\boldsymbol{\xi} h, \boldsymbol{\xi}, t_{n}\right)=\frac{h}{2}\left[\Omega\left(\boldsymbol{r}_{i j}, \boldsymbol{\xi}, t_{n}+h\right)+\Omega\left(\boldsymbol{r}_{i j}-\boldsymbol{\xi} h, \boldsymbol{\xi}, t_{n}\right)\right],
$$

where $\boldsymbol{r}_{i j}$ is the center of cell interface between cell $i$ and cell $j$.

By introducing two auxiliary distribution functions $\bar{f}$ and $\bar{f}^{+}$expressed as

$$
\bar{f}=f-\frac{h}{2} \Omega_{B}=\frac{2 \tau+h}{2 \tau} f-\frac{h}{2 \tau} f^{e q}
$$




$$
\bar{f}^{+}=f+\frac{h}{2} \Omega_{B}=\frac{2 \tau-h}{2 \tau+h} \bar{f}+\frac{2 h}{2 \tau+h} f^{e q}=\frac{2 \tau-h}{2 \tau+\Delta t} \tilde{f}+\frac{3 h}{2 \tau+\Delta t} f^{e q},
$$

the Eq.(16) can be transformed into the following form

$$
\bar{f}\left(\boldsymbol{r}_{i j}, \boldsymbol{\xi}, t_{n+1 / 2}\right)=\bar{f}^{+}\left(\boldsymbol{r}_{i j}-\boldsymbol{\xi} h, \boldsymbol{\xi}, t_{n}\right) .
$$

According to Eqs.(22) and (25), the density and velocity can also be obtained from $\bar{f}$, i.e.,

$$
n=\int \bar{f} d \boldsymbol{\xi}, \quad n \boldsymbol{u}=\int \boldsymbol{\xi} \bar{f} d \boldsymbol{\xi}
$$

Meanwhile, $\bar{f}^{+}$and $\tilde{f}^{+}$satisfy the following relationship, which will be used to evaluate the $\tilde{f}^{+}$ in Eq.(21)

$$
\tilde{f}^{+}=\frac{4}{3} \bar{f}^{+}-\frac{1}{3} \tilde{f}
$$

Once $\bar{f}\left(\boldsymbol{r}_{i j}, \boldsymbol{\xi}, t_{n+1 / 2}\right)$ is evaluated from Eq.(27), the original distribution function $f\left(\boldsymbol{r}_{i j}, \boldsymbol{\xi}, t_{n+1 / 2}\right)$ at interface center $\boldsymbol{r}_{i j}$ can be calculated according to the relationship between $\bar{f}$ and $f$ in Eq.(25), after which the micro flux is obtained according to Eq.(18). Thus, the main task is to construct the $\bar{f}^{+}\left(\boldsymbol{r}_{i j}-\boldsymbol{\xi} h, \boldsymbol{\xi}, t_{n}\right)$ in Eq.(27), and to obtain $\bar{f}\left(\boldsymbol{r}_{i j}, \boldsymbol{\xi}, t_{n+1 / 2}\right)$ consequently.

Generally, $\bar{f}^{+}\left(\boldsymbol{r}_{i j}-\boldsymbol{\xi} h, \boldsymbol{\xi}, t_{n}\right)$ can be expanded around $\bar{f}^{+}\left(\boldsymbol{r}_{i j}, \boldsymbol{\xi}, t_{n}\right)[31]$ or $\bar{f}^{+}\left(\boldsymbol{r}_{i}, \boldsymbol{\xi}, t_{n}\right)[33]$ by assuming a linear relationship. Considering a significant density oscillation may occur in a nanoscale dense fluid system, we will expand it around the cell center value and employ the van Leer limiter [54]. The linear relationship and limiter can be expressed as

$$
\bar{f}^{+}\left(\boldsymbol{r}_{i j}-\boldsymbol{\xi} h, \boldsymbol{\xi}, t_{n}\right)=\bar{f}^{+}\left(\boldsymbol{r}_{j}, \boldsymbol{\xi}, t_{n}\right)+\left[\left(\boldsymbol{r}_{i j}-\boldsymbol{\xi} h\right)-\boldsymbol{r}_{j}\right] \cdot \boldsymbol{\gamma}_{j},\left(\boldsymbol{r}_{i j}-\boldsymbol{\xi} h\right) \in V_{j},
$$

where $\gamma_{j}$ is the corresponding slop at the cell $V_{j}$. Taking the component in the $x$ direction for example, the slope can be written as

$$
\gamma_{j, x}=\left[\operatorname{sign}\left(s_{1}\right)+\operatorname{sign}\left(s_{2}\right)\right] \frac{\left|s_{1}\right|\left|s_{2}\right|}{\left|s_{1}\right|+\left|s_{2}\right|},
$$

where 


$$
s_{1}=\frac{\bar{f}^{+}\left(x_{j}\right)-\bar{f}^{+}\left(x_{j-1}\right)}{x_{j}-x_{j-1}}, s_{2}=\frac{\bar{f}^{+}\left(x_{j+1}\right)-\bar{f}^{+}\left(x_{j}\right)}{x_{j+1}-x_{j}} .
$$

Up to now, all the variables needed for the evolution equation, i.e., Eq.(21) are solved, where the external force term $\boldsymbol{G}$ is not included. The Strang splitting algorithm [51] will be introduced below on how to couple the external force term $\boldsymbol{G}$ into the standard DUGKS evolution $[52,53]$ as described above.

\subsection{Strang splitting method for external force}

In the Strang splitting method, a half time step integration is implemented on distribution functions before and after the standard DUGKS procedure, which is called pre-forcing and post-forcing step, respectively. The pre-forcing step, the standard DUGKS and the post-forcing step can be respectively written as

$$
\begin{gathered}
\partial_{t} f=0.5 \boldsymbol{G}, \\
\partial_{t} f+\boldsymbol{\xi} \cdot \nabla_{r} f=\Omega_{B}, \\
\partial_{t} f=0.5 \boldsymbol{G} .
\end{gathered}
$$

The evolution from time $t_{n}$ to $t_{n+1}$ in the Strang splitting algorithm can be seen in Figure 1 .

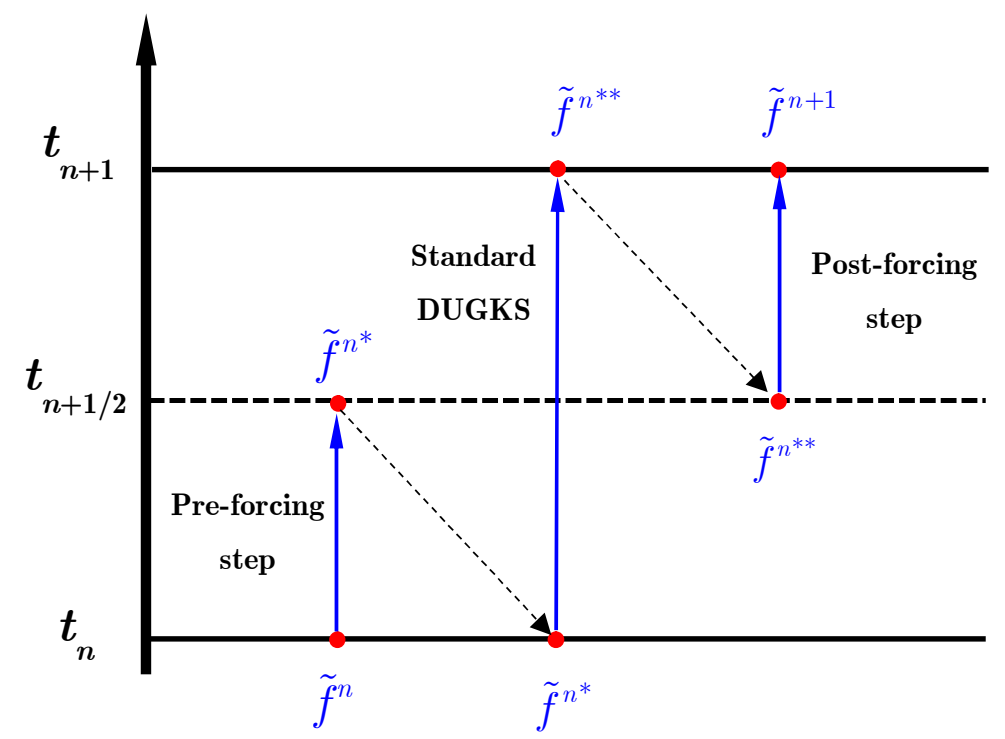


Figure 1: The evolution procedure from time $t_{n}$ to $t_{n+1}$ in the Strang splitting method. $f^{n^{*}}$ and $f^{n^{* *}}$ represent the solutions from the pre-forcing step and the standard DUGKS, respectively.

In the pre-forcing and/or post-forcing step, integrate Eqs.(33) and/or (35) over a time step $\Delta t$, we have

$$
f^{*}=f+\frac{\Delta t}{2} \boldsymbol{G}(n, \boldsymbol{u})
$$

where $f^{*}$ is a solution from the pre-forcing or post forcing step.

According to Eq.(19), we have the following relations

$$
\begin{gathered}
\tilde{f}=\frac{2 \tau+\Delta t}{2 \tau} f-\frac{\Delta t}{2 \tau} f^{e q}(n, \boldsymbol{u}), \\
\tilde{f}^{*}=\frac{2 \tau+\Delta t}{2 \tau} f^{*}-\frac{\Delta t}{2 \tau} f^{e q}\left(n^{*}, \boldsymbol{u}^{*}\right),
\end{gathered}
$$

where the density and velocity can be calculated as

$$
n^{*}=n, \boldsymbol{u}^{*}=\boldsymbol{u}+0.5 \boldsymbol{G} \Delta t
$$

Coupling Eqs.(36), (37) and (38), $\tilde{f}^{*}$ can be calculated from $\tilde{f}$ by the following equation

$$
\tilde{f}^{*}=\tilde{f}+\frac{\Delta t}{2 \tau}\left[f^{e q}(n, \boldsymbol{u})-f^{e q}\left(n^{*}, \boldsymbol{u}^{*}\right)\right]+\frac{(2 \tau+\Delta t) \Delta t}{4 \tau} \boldsymbol{G}(n, \boldsymbol{u}) .
$$

\subsection{Relaxation time}

Based on the LAD method [55], the relaxation time $\tau$ in the collision operator $\Omega_{\mathrm{B}}$ of Eq.(2) is determined by

$$
\tau=\frac{\mu(\bar{n})}{n k_{B} T}
$$

where $k_{\mathrm{B}}$ is the Boltzmann constant, $\mu(\bar{n})$ is the viscosity of homogeneous dense fluid evaluated at the LAD $\bar{n}$ expressed as [23] 


$$
\mu(\bar{n})=\frac{5.0}{16 \sigma^{2}} \sqrt{\frac{k_{B} T}{\pi}} \bar{n} V_{0}\left(Y^{-1}+0.8+0.7614 Y\right)
$$

where the parameter $Y$ and the radial distribution function $\chi$ is calculated by [44-46]

$$
Y=n V_{0} \chi(\bar{n}), \quad \chi(\bar{n})=\frac{1-0.5 \eta}{(1-\eta)^{3}}, \quad \eta=\frac{\bar{n} V_{0}}{4}, \quad V_{0}=\frac{2 \pi \sigma^{3}}{3}
$$

\subsection{Boundary condition}

In the kinetic models, appropriate boundary conditions should be given for the distribution functions at the solid walls [31]. The surface slip significantly depends on the relative strength between fluid-wall and fluid-fluid interactions, which can be characterized by the ratio of energy parameter $\varepsilon_{w f}$ to $\varepsilon_{f f}[56]$. With the decrease of the ratio $\varepsilon_{w f} / \varepsilon_{f f}$, the boundary transforms from wetting to non-wetting, and the slip increases correspondingly. In this study, the bounce-back boundary condition is employed for a no-slip boundary condition to simulate the wetting cases, where gas molecules adsorb on the wall and form an adsorption layer when hitting the solid molecules, rather than the usual diffuse or specular reflection. The slip boundary condition for the non-wetting cases can also be achieved by the bounce back boundary condition with a slip velocity, which is determined by the fluid-solid interactions. Note that, even in the non-wetting case, weak adsorption layers may form near the wall, as shown in Figure $8 b$. In this paper, we mainly focus on the wetting case with a no-slip boundary condition, and the detailed bounce back scheme can be referred to Guo et al. [31].

\subsection{Algorithm}

The standard procedure of the DUGKS from time $t_{n}$ to $t_{n+1}$ is the same as the previous study [33]. The difference lies in how to couple the external force $\boldsymbol{G}$ by Strang splitting technique with the standard DUGKS $[52,53]$, as shown in Figure 1. Detailed computational 
procedures can be summarized in the following steps:

(1) Pre-forcing step calculation with a half time step $h=\Delta t / 2$.

(1) Determine the local average density $\bar{n}$, which can be referred to Bitsanis et al. [57], Tarazona [45] or Vanderlick et al.. [29];

(2) Calculate the radial distribution function $\chi$ according to Eq.(43);

(3) Compute the gradients of the radial distribution function and local average density according to Eqs.(6) and (7), respectively;

(4) Coupling the force term $\boldsymbol{G}$ into the Strang splitting algorithm according to Eq.(40).

(2) The standard DUGKS evolution from time $t_{n}$ to $t_{n+1}$.

(1) Calculate $\bar{f}^{+}$from $\tilde{f}$ at cell interface according to Eq. (26);

(2) Compute the gradient of $\bar{f}^{+}$in each cell according to Eq.(31);

(3) Calculate the distribution function $\bar{f}^{+}$at $\left(\boldsymbol{r}_{i j}-\boldsymbol{\xi} h\right)$ according to Eq.(30);

(4) Determine the distribution function $\bar{f}$ at cell interface and time $t_{n+1 / 2}$ according to Eq.(27);

(5) Calculate the conserved flow variables from $\bar{f}$ according to Eq.(28);

(6) Determine the original distribution function $f$ at cell interface and time $t_{n+1 / 2}$ from $\bar{f}\left(\boldsymbol{r}_{i j}, \boldsymbol{\xi}, t_{n+1 / 2}\right)$ and $f^{e q}\left(\boldsymbol{r}_{i j}, \boldsymbol{\xi}, t_{n+1 / 2}\right)$ according to Eq.(25);

(7) Calculate the flux $\boldsymbol{F}^{n+1 / 2}$ through each cell interface from $f^{n+1 / 2}$ according to Eq.(18);

(8) Determine $\tilde{f}^{+}$at cell center and time $t_{n}$ according to Eq.(29);

(9) Update the cell-averaged $\tilde{f}$ in each cell from $t_{n}$ to $t_{n+1}$ according to Eq.(21);

(3) Post-forcing step (the same as the pre-forcing step).

\section{Model validation}

In this part, the static fluid structure and flow behaviors of dense fluid systems confined between two plates with a separation of $H$ at the nanometer scale, as sketched in Figure 2, are studied. For such a system, the fluid molecular size can no longer be neglected comparing to the channel width $H$, which means that the Boltzmann equation fails under this circumstance. 


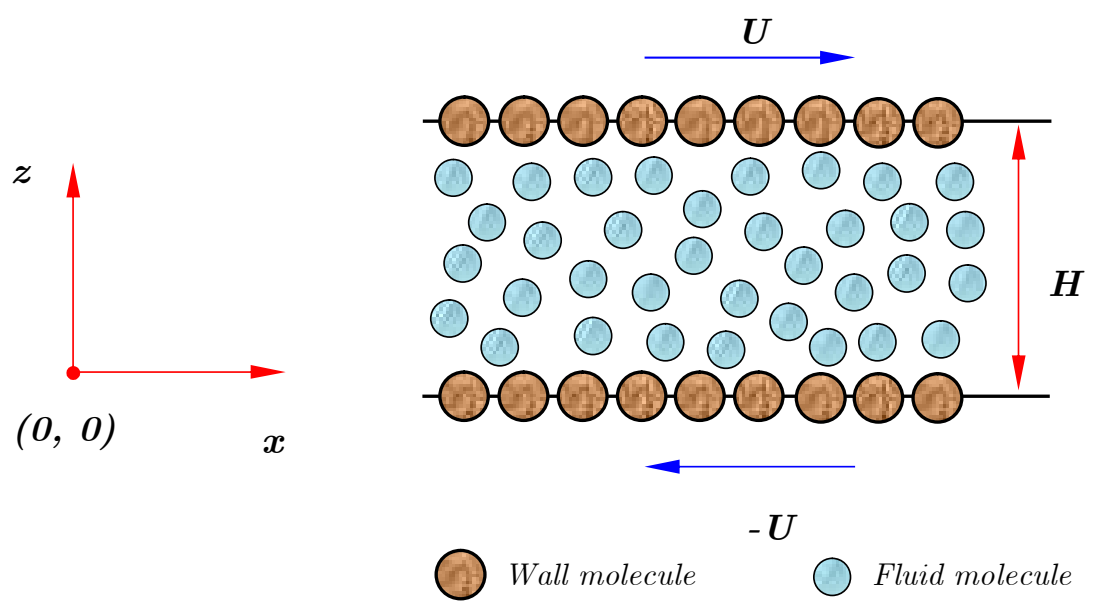

Figure 2: Fluid molecules confined between two paralleled plates at the nanometer scale.

\subsection{Static fluid structure}

The equilibrium structure of the LJ fluids with three different channel widths are first tested. In the simulation, the $10-4-3 \mathrm{LJ}$ potential is exerted on the fluid molecules by the top and bottom plates, while the $12-6$ LJ potential is employed for fluid molecular interactions. The channel widths and pore averaged density, which is defined as $n_{0}=\int_{0}^{H} n(y) d y / H$, are displayed in Figure 3, while the fluid system temperature is taken as $T_{r}=1.2$ for all the three cases. Meanwhile, the solid-fluid energy parameter $\varepsilon_{w f}$ equals fluid-fluid energy parameter $\varepsilon_{f f}$, meaning the strength of solid-fluid interactions and fluid-fluid interactions is approximately the same.

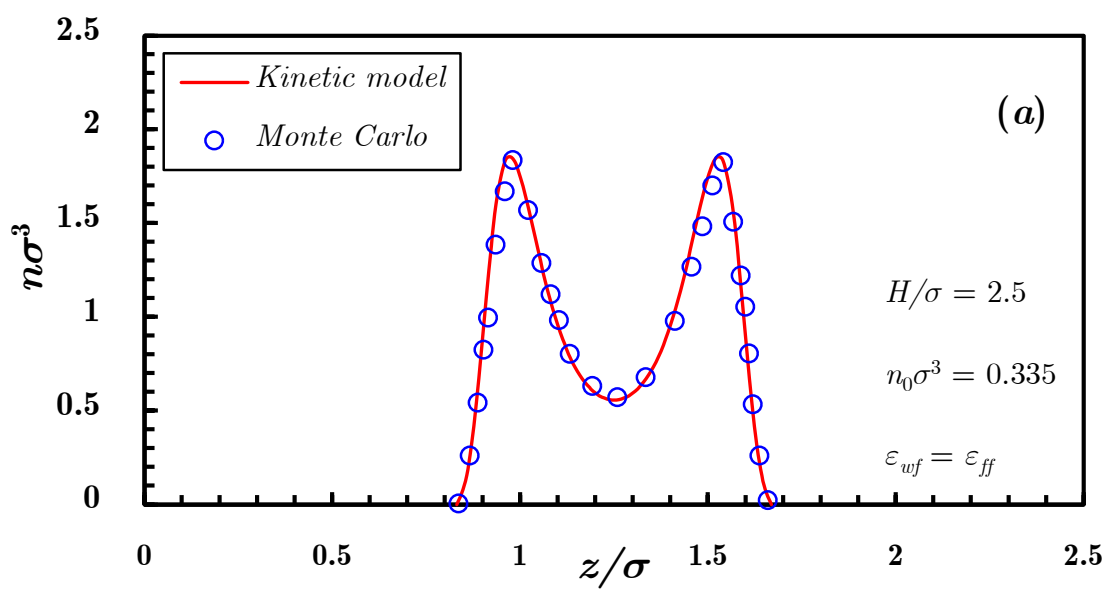



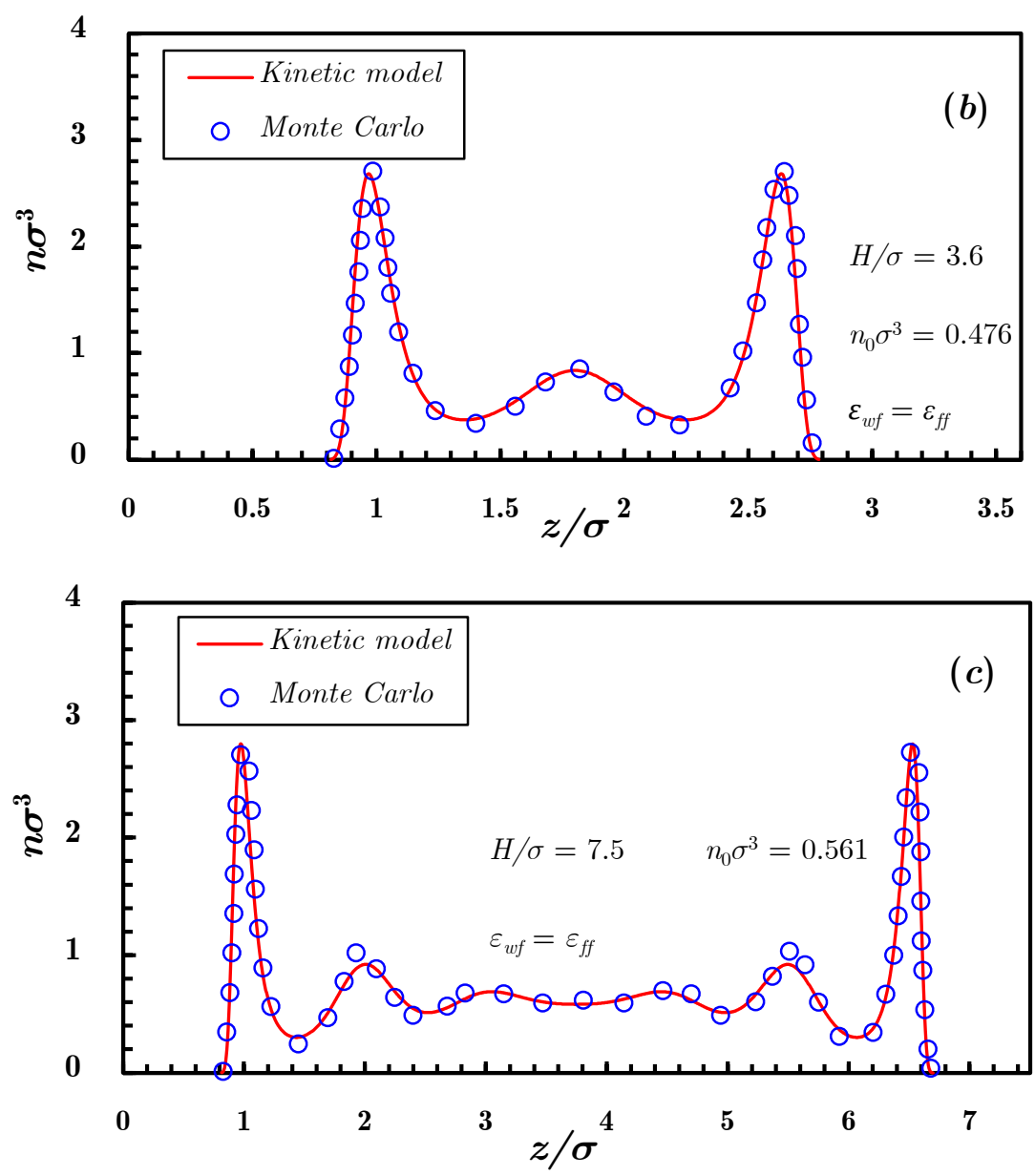

Figure 3: Density distributions of LJ fluids confined between two paralleled plates with $10-4-3 \mathrm{LJ}$ potential at the temperature of $T=1.2 \varepsilon_{f f} / k_{B} ;$ Monte Carlo results can be referred to [58]

As shown in Figure 3, the density profiles in all the cases oscillates significantly across the channel due to the combined effects of external wall potential, volume exclusion effects and long-range intermolecular interactions. The unique density structures agree well with the Monte Carlo results [58] in all the three cases, including the magnitudes and locations of peaks as well as their oscillation tendency. No bulk regions appear for these narrow channels, i.e., $H$ $=2.5 \sigma$ (Figure $3 a)$ and $H=3.6 \sigma$ (Figure $3 b$ ), while the fluid will become homogeneous near the center region with the increase of channel width (Figure $3 c$ ). Due to the strong repulsion from the solid molecules, it is hard for fluid molecules to approach the boundary and there will be a vacuum layer between the first fluid layer and the wall, with the thickness equaling to $\delta$. The 
capability of capturing the critical changes in fluid structure reveals the applicability of the present DUGKS in predicting the fluid structure induced by external wall potential and fluid molecular interactions.

\subsection{Dynamic behaviors}

\subsubsection{Couette flow}

The second test case is the Couette flow, with the top and bottom plates moving with a velocity of $U=0.5 \sqrt{k_{B} T / m}$ in the $x$ and $-x$ directions, respectively, as shown in Figure 2. In the computation, the grid size in the $y$ direction is set to be $\Delta y=0.01 \mathrm{H}$, which is fine enough to produce grid independent solutions. Meanwhile, 8 Gauss-Hermit discrete velocities distributed in $\left[-4 \sqrt{2 k_{B} T / m}, 4 \sqrt{2 k_{B} T / m}\right]$ are used to discretize the velocity space in each direction. Although the local Knudsen number can be very high, as will be discussed later, the set of $8 \times 8$ discretized velocities is sufficient to capture the non-equilibrium effects of the current problem, as we have tested. The CFL number is set to be 0.1 . The fluid is confined between two $10-4$ walls with a separation of $H=7.178 \sigma$, in which the pore average density of the confined fluids is $n_{0}=0.593 \sigma^{-3}$ with the temperature of $T_{r}=1.0$.

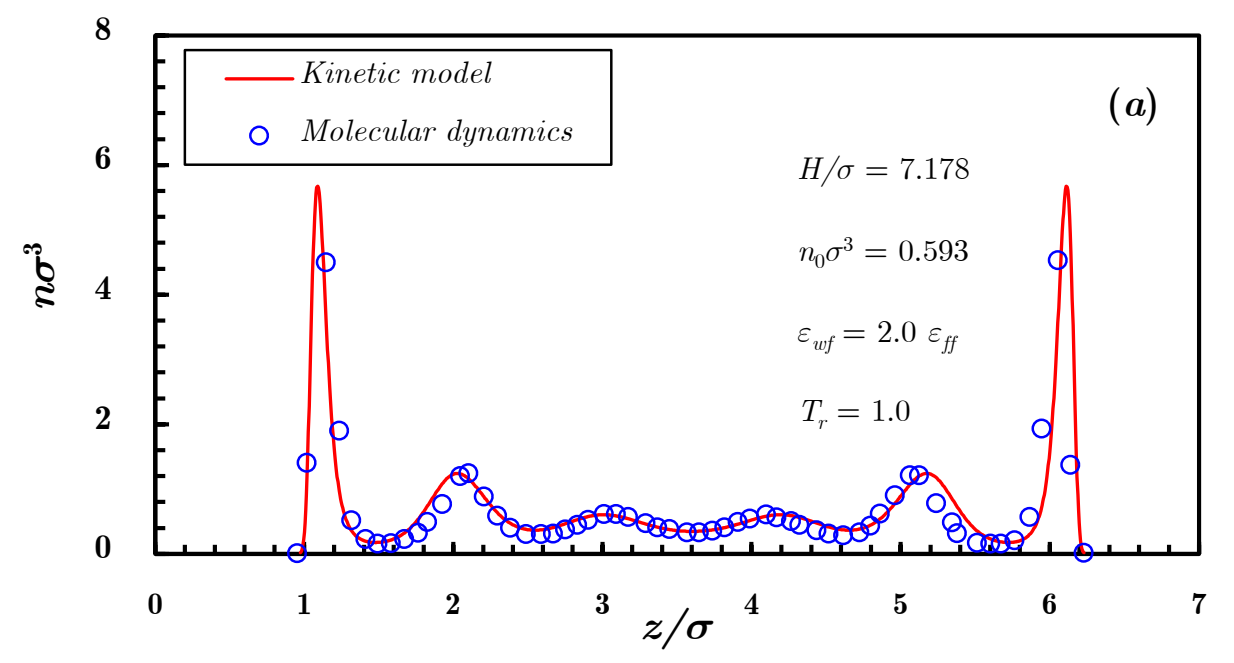




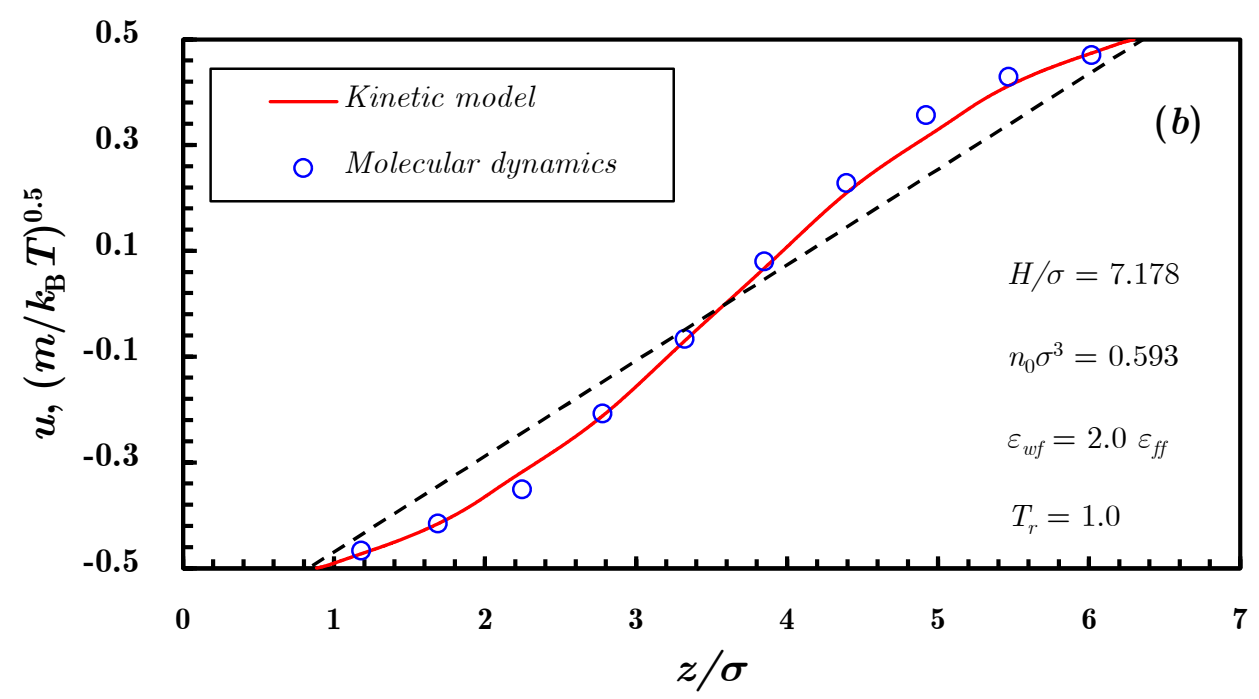

Figure 4: Density (a) and velocity $(b)$ profiles of Couette flow for LJ fluids in confined plates with $10-4$ potential.

MD results can be referred to [55]. The dotted line in the bottom panel represents the linear distribution of the velocity profile predicted by the conventional hydrodynamic model, which ignores the competition between the fluid-fluid and fluid-solid interactions.

The static structure and dynamic behaviors of the LJ fluids for Couette flow are also satisfactorily captured by the current DUGKS comparing to the MD results [55], as shown in Figure 4. Three adsorption layers with decreasing intensity are observed in the vicinity of each wall, while there is no obvious bulk region near the center of the channel (Figure 4a). As shown in Figure $4 b$, the velocity distribution of Couette flow deviates from linearity, as a result of fluid inhomogeneity induced by the competition of the wall-fluid and fluid-fluid interactions. Thus, it is essential to take the wall potentials and fluid molecule interactions into account at the nanometer scale, which greatly affects the density (Figure 4a) and velocity (Figure 4b) distributions across the channel.

The effects of flow channel widths on density and velocity distribution of Couette flow are also investigated. In the simulation, the top and bottom plates move with a velocity of 
$U=0.01 \sqrt{k_{B} T / m}$ in opposite directions. The confined fluids, with an averaged density of $n_{0}$ $=0.561 \sigma^{-3}$ at the temperature of $T_{r}=1.2$, are simulated with the $10-4-3$ potentials from the top and bottom walls, respectively. Meanwhile, the energy parameter of the wall-fluid interactions $\varepsilon_{w f}$ is four times of that for fluid-fluid interactions $\varepsilon_{f f}$. As is shown in Figure 5, there are two obvious adsorption layers in the vicinity of each wall, after which a slightly third adsorption layer occurs. All the three adsorption layers coincide together, indicating a similar effect is exerted on fluid molecules from the wall. The bulk region increases with the channel width $H$ increasing. The velocity profile tends to be linearly distributed across the channel with the increase of the channel widths $H$ (Figure $5 b$ ). This is because the inhomogeneity of the fluid system becomes weaker in larger scale systems. It also means that there is a critical value for the channel width, over which the inhomogeneity of the system can be ignored. The determination of the critical value will be studied in the future.

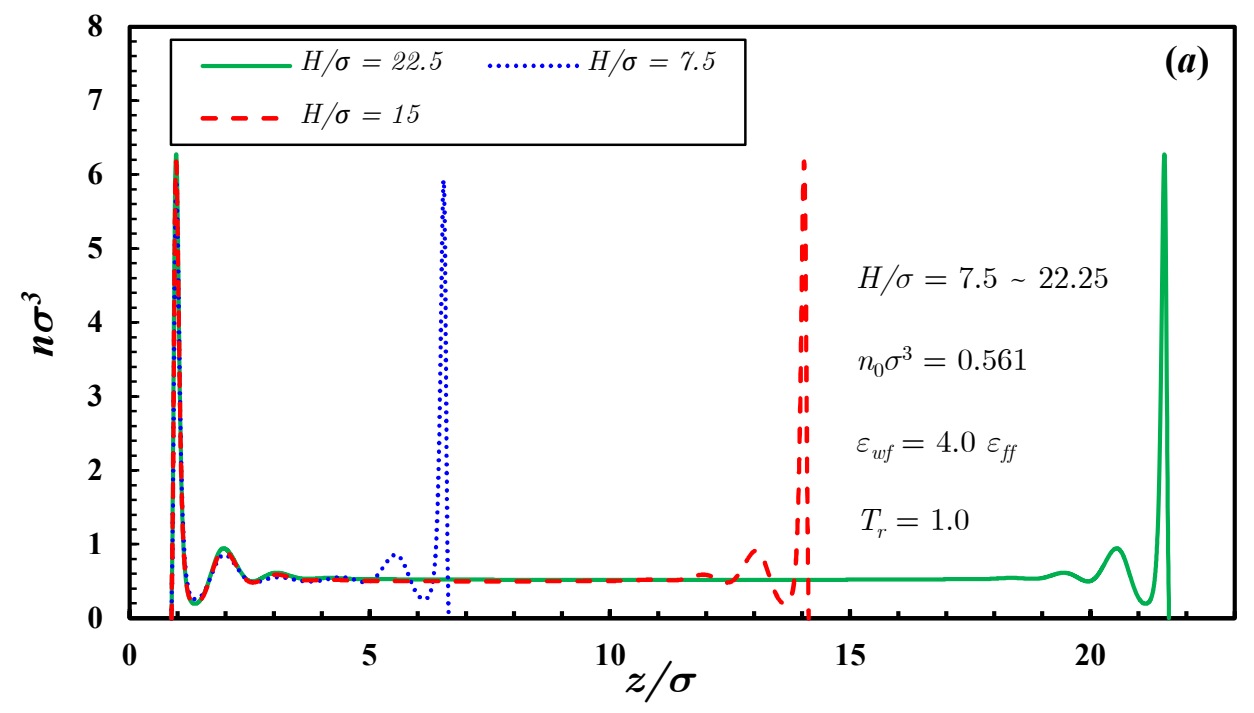




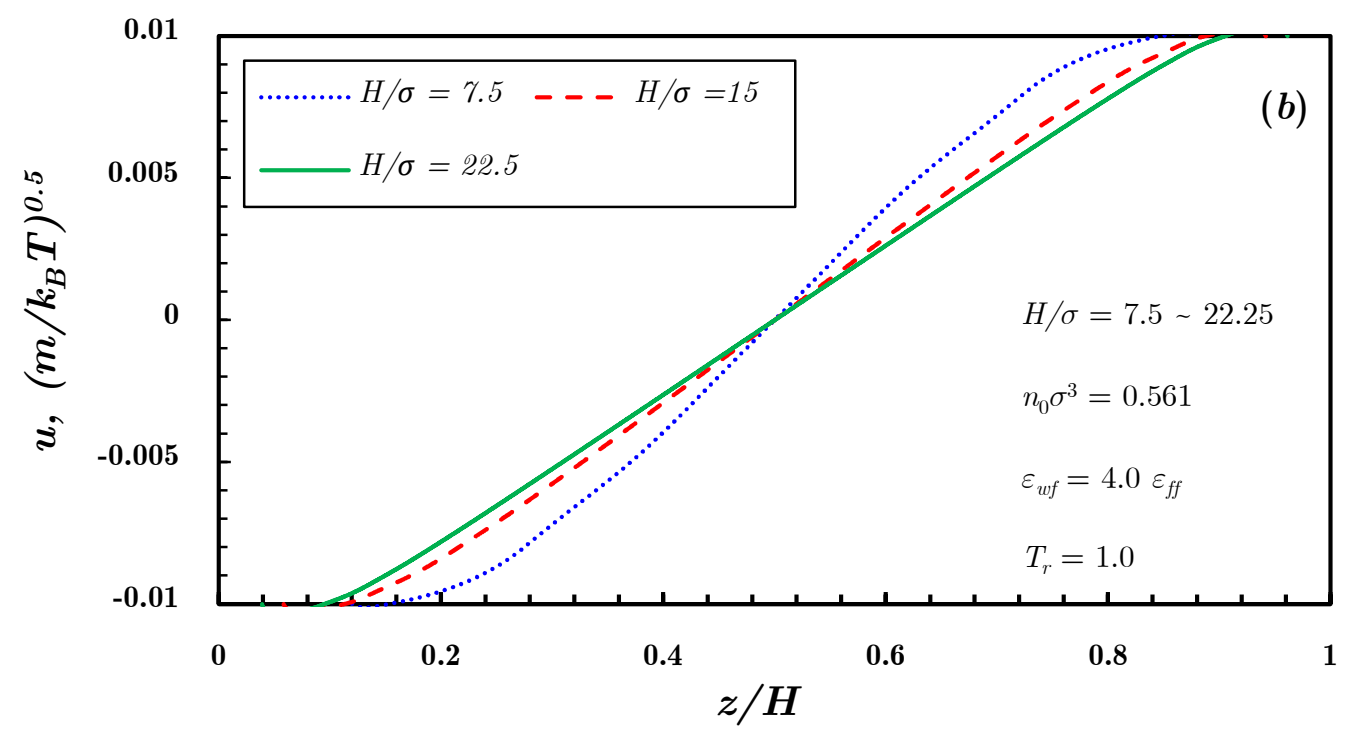

Figure 5: Effects of channel width on density $(a)$ and velocity $(b)$ profiles of Couette flow with the $10-4-3$ wall potential

\subsubsection{Poiseuille flow}

Finally, we take the Poiseuille flow as our third test case. Adopting the same parameters as presented in Figure $3 b$ and exerting a driving force of $G_{x}=0.02 \varepsilon_{f f} / \sigma$ in the $x$ direction, the previous static problem transforms into the Poiseuille flow. According to the dense gas theory [23], $n V_{0}$ in Eq.(43) is a parameter reflecting the denseness of the fluids. In our simulation, the pore averaged density is kept constant at $n_{0}=0.476 \sigma^{-3}$, while $V_{0}$ is changed to control the denseness of the confined fluids. 

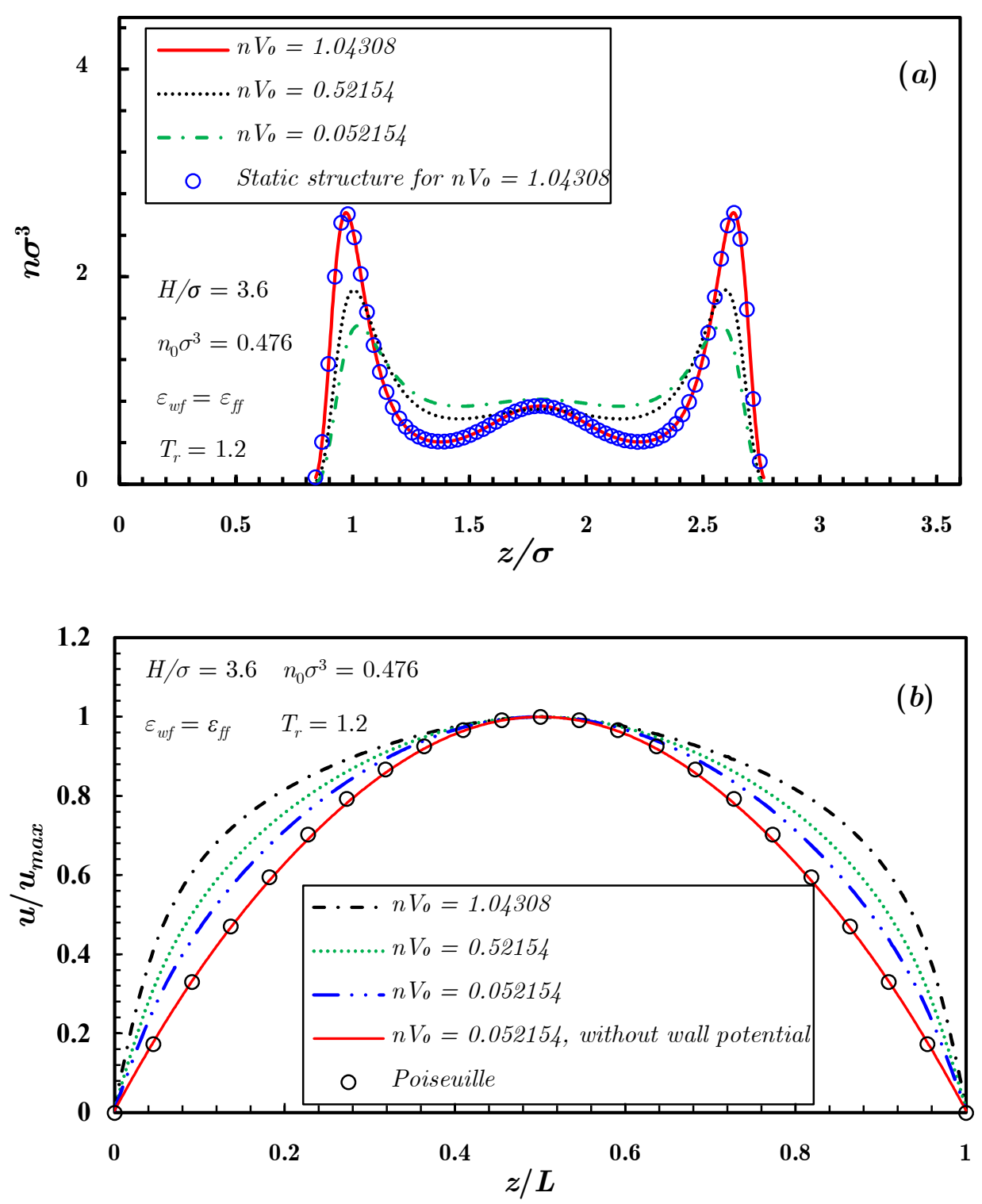

Figure 6: Density $(a)$ and velocity $(b)$ profiles for Poiseuille flow, $n V_{0}$ is used to control the dense degree, and $L$ $=H-2 \delta$.

As we can see from Figure $6 a$, the flow does not affect the density distribution across the channel, and the denser the fluids, the more oscillatory the density distribution. In Figure $6 b$, the velocity is normalized by the maximum velocity $u_{\max }$ and the distance is normalized by effective flow domain length $L=H-2 \delta$. As shown in Figure $6 b$, the velocity profiles at the nanometer scale (for $n V_{0} \approx 1.04308$ ) also deviate from the analytical solution of the Poiseuille flow governed by the classical Navier-Stokes equation significantly, due to the 
combined effects of wall-fluid and fluid-fluid interactions. The velocity tends to the parabolic as the fluid becomes more dilute with decreasing $n V_{0}$, and recovers exactly the analytical solution when $n V_{0}$ decreases to 0.052154 , without considering the effects of wall potential.
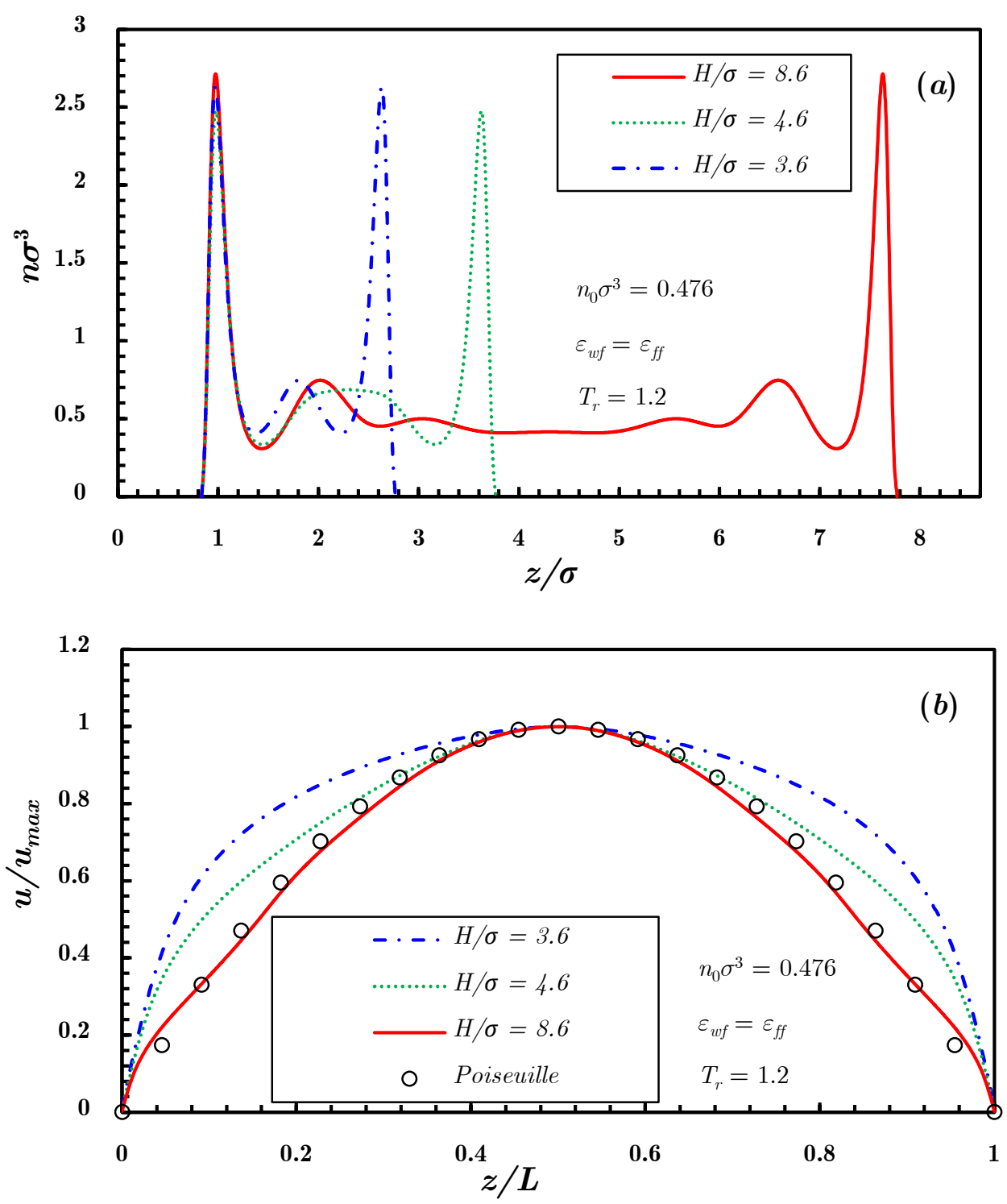

Figure 7: Density $(a)$ and velocity $(b)$ profiles under different channel-width conditions for Poiseuille flow, where $L=H-2 \delta$.

The above Poiseuille flow is also studied under different channel-width conditions. As shown in Figure $7 a$, the density fluctuates across the whole flow domain as $H=3.6 \sigma$, while a bulk region may occur with the increase of channel width. This is because the competition 
between the solid-fluid and fluid-fluid interactions becomes weaker near the center region of a larger channel, and the influences from the wall are limited. Similarly, the velocity profile approaches to the Navier-Stokes solution with the increase of the channel width, which further supports our assessment that the fluid system becomes more homogeneous in large systems.
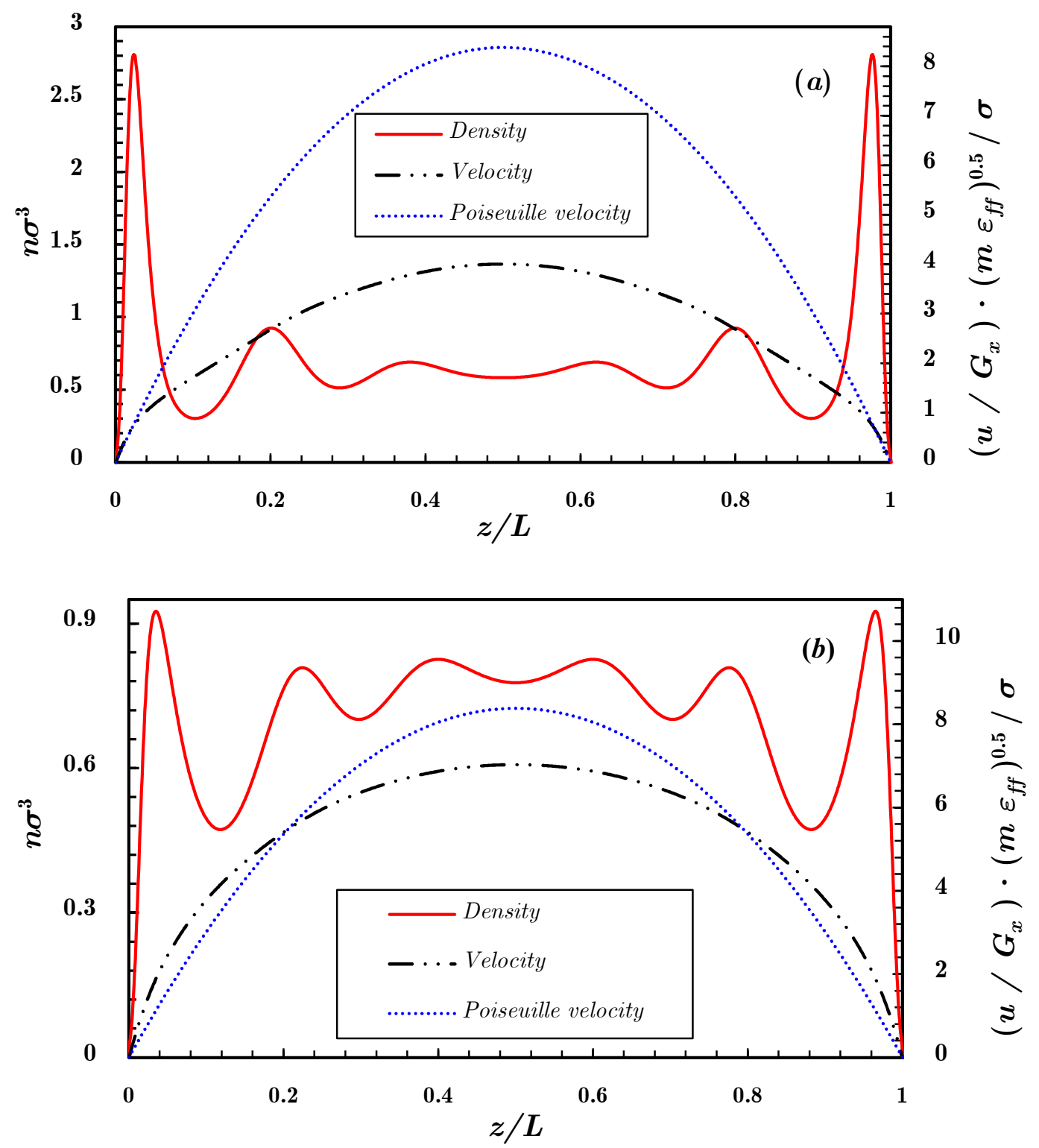

Figure 8: The density and velocity profiles for Poiseuille flow in confined $10-4-3$ LJ channels at the temperature of $T_{r}=1.2$ : $(a)$ wetting case, $\varepsilon_{w f} / \varepsilon_{f f}=1.0 ;(b)$ non-wetting case, $\varepsilon_{w f} / \varepsilon_{f f}=0.25$. The velocity profiles were normalized by the external driving force $G_{x}$. The pore averaged density is $0.561 \sigma^{-3}$ and the channel with is $7.5 \sigma$. The locations were normalized by the length of the flow domain $L=H-2 \delta$. 
The density and velocity profiles for Poiseuille flow with the wall separation of $H=7.5 \sigma$ at the temperature of $T_{r}=1.2$ are shown in Figure 8, where the velocity is normalized by the external driving force $G_{x}$ and the location is normalized by the flow domain $L$. For the wetting case $\left(\varepsilon_{w f} / \varepsilon_{f f}=1.0\right)$, fluid molecules accumulate near the wall due to the strong solid-fluid interactions, and the velocity is much smaller than the analytical Poiseuille solution across the channel. When the ratio $\varepsilon_{w f} / \varepsilon_{f f}$ decreases to 0.25 , the walls become non-wetting, and the density oscillation becomes much flatter, comparing to the wetting case in Figure $8 a$. However, the adsorption layers near the wall still exist, but with much smaller magnitude. Meanwhile, the velocity becomes much higher than that under wetting conditions, implying a significant effect of wettability on flow velocity.

We want to point out that the computational efficiency of the DUGKS is much higher than that of the MD simulations. In one of our test cases of the Poiseuille flow under the same working condition, the computing time of the DUGKS running with single core is about ten minutes, while it is more than seven hours of MD simulations running with 24 cores to obtain satisfactorily stable results.

\subsubsection{Multiscale characteristics of dense fluid system}

The Knudsen number is commonly used as a key criterion number in multiscale analysis from continuum flow to free molecular flow in rarefied gas dynamics. However, fluid molecular movements are frequently disrupted by the walls or other molecules due to the small dimension of the flow path or the dense arrangements of the fluid molecules in the case we study, which means that the molecules cannot move freely. Thus, the Knudsen number in this paper is actually the effective Knudsen number, which is borrowed from rarefied gas dynamics 
for the current dense fluid system. According to the difference in defining the characteristic lengths, the average effective Knudsen number $K n$ and local effective Knudsen number $K n^{*}$ can be defined as

$$
K n=\frac{\lambda}{H}, \quad K n^{*}=\frac{\lambda}{\rho /|\nabla \rho|}
$$

where $\lambda$ is the gas mean free path, and $\rho /|\nabla \rho|$ is the local characteristic length. According to dense gas theory [4,23], the mean free path is determined by

$$
\lambda=\frac{1}{\sqrt{2} n \pi \sigma^{2} \chi} .
$$

On one hand, the average effective Knudsen number is calculated as $K n=0.06$, according to Eq.(44) and the parameters in Figure 3b, from which we may deduce that the non-equilibrium effects are not very obvious; on the other, the density varies sharply near the wall, resulting an enormous local characteristic length, where the local effective Knudsen number can be as high as 106.976. Beyond that, it is almost smaller than 0.5 near the center region, and even as small as 0.00058 in certain places. The violent fluctuation of the local effective Knudsen number, as shown in Figure 9, implies the strong inhomogeneity of the dense fluid system across the channel.

Meanwhile, the mechanism of rarefaction effect in a dense fluid system may be very different from that in a rarefied one. For the Poiseuille flow of rarefied gases, the rarefaction effect mainly occurs at gas-solid interface showing as a velocity slip, which is attributed to the infrequent collisions between the gas and solid molecules. However, the density is strongly inhomogeneous in dense fluid systems, which means the amount of gas molecules between the adjacent adsorption layers is very small, even close to the vacuum, where the rarefaction effect 
may occur, rather than at the fluid-solid interface. The detailed mechanism of rarefaction effect in dense fluid system is very complicated, which needs deeper investigation in the future. According to our test cases, the velocity profile does not converge using lower-order Gauss-Hermite quadrature of velocity space, for example, 4 discretized velocity points in $x$ and $y$ direction, respectively. This phenomenon suggests rarefaction effects do exist in dense fluid system and the system is a multiscale one. The numerical results demonstrate the capability of the DUGKS in simulating fluid flows for all the effective Knudsen numbers.

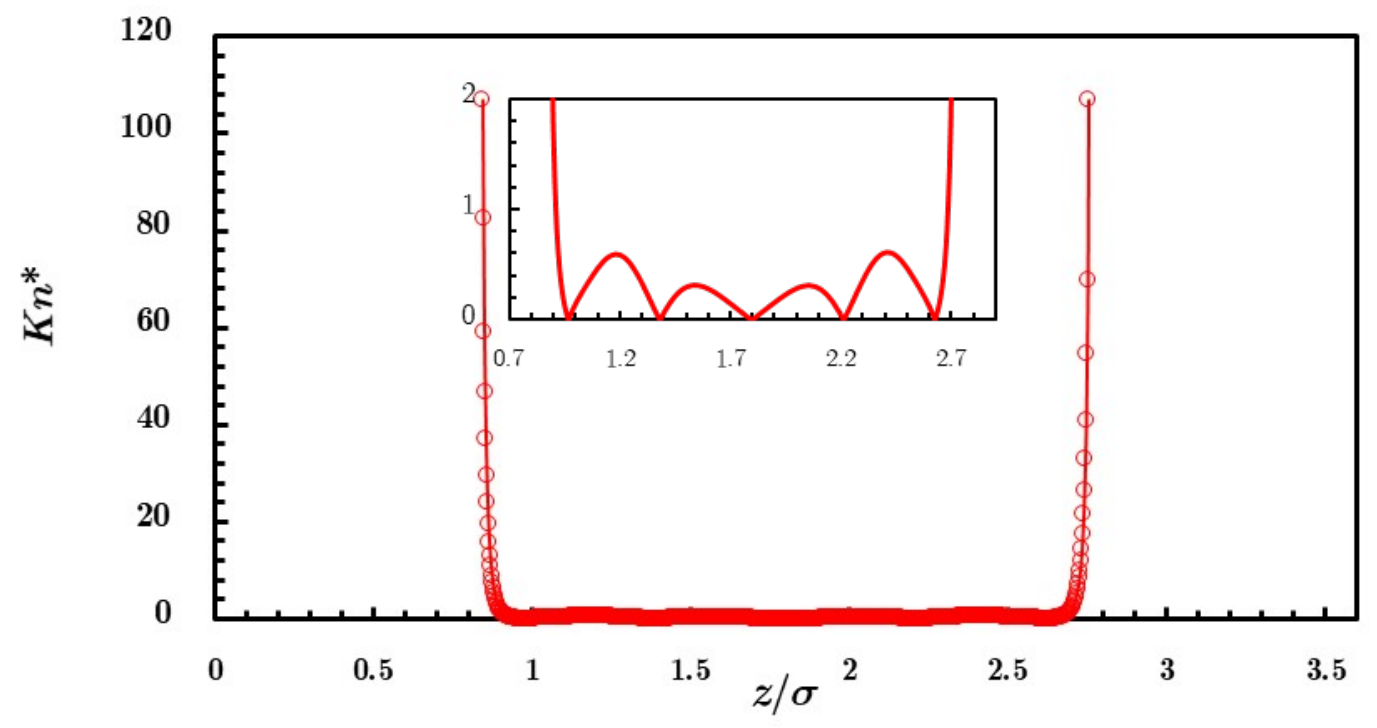

Figure 9: The variation of local effective Knudsen number across the channel with the $10-4-3$ potential, where $H / \sigma=3.6, n \sigma^{3}=0.476, T_{r}=1.2$ and $\varepsilon_{w f}=\varepsilon_{f f}$.

\section{Conclusions}

It is a challenging task to capture the non-equilibrium effects of dense fluid flows at the nanometer scale. In this paper, the DUGKS is extended to strongly inhomogeneous fluid systems, where the external wall potential, volume exclusion effects and long-range intermolecular attractions are simultaneously taken into account. These non-equilibrium 
effects are coupled into a unified force term, which is conveniently incorporated into the DUGKS by the Strang-splitting method. The time step of the DUGKS is not limited by particle collision time in multiscale flow regimes, which indicates that the DUGKS is an ideal tool to simulate dense fluid flow dynamics, since the effective Knudsen number may vary significantly under different conditions.

The static fluid structures and dynamic flow behaviors agree well with Monte Carlo simulation and/or MD results, which proves the capability of our model in capturing the non-equilibrium effects of dense fluid systems at the nanometer scale where the local effective Knudsen number can vary from the order of 0.0001 to the order of 100 . There will be a vacuum between the first fluid layer and the wall due to the strong repulsion, while several adsorption layers may occur due to the competition of solid-fluid and fluid-fluid interactions. It is also found the density distribution across the channel is not affected by the fluid flow. The velocity profile of the Couette flow deviates from the linear distribution, while the velocity profile of Poiseuille flow deviates from the Navier-Stokes solution significantly as a result of inhomogeneous nature of the dense fluids at the nanometer scale.

In our future work, more practical boundary conditions will be considered in establishment, which may serve as a powerful tool between the connections of MD simulation and the Navier-Stokes equation.

\section{Acknowledgments}

This work was supported by the National Science Foundation of China (Grant No. 51836003). Wang and Zhang would like to thank the financial support from the UK's Engineering and Physical Sciences Research Council under grant number EP/M021475/1. 
Helpful discussions with Dr. Songze Chen and valuable advice from him are highly appreciated. 


\section{References:}

[1] L. Wu, Y. Zhang, and J.M. Reese, J Comput Phys 303, 66 (2015).

[2] A.V. Raghunathan, J.H. Park, and N.R. Aluru, J Chem Phys 127, 174701 (2007).

[3] J. Badur, P. Ziółkowski, S. Kornet, T. Kowalczyk, K. Banaś, M. Bryk, P.J. Ziółkowski, and M. Stajnke, J Theor App Mech-Pol, 329 (2018).

[4] L. Wu, H. Liu, J.M. Reese, and Y. Zhang, J Fluid Mech 794, 252 (2016).

[5] L. Zhang, S. Baochao, Z. Yulong, and G. Zhaoli, Int J Heat Mass Tran 139, 144, 1 (2019).

[6] C. Wang, Y.V. Kaneti, Y. Bando, J. Lin, C. Liu, J. Li, and Y. Yamauchi, Mater Horiz 5, 394 (2018).

[7] M.A. Shannon, P.W. Bohn, M. Elimelech, J.G. Georgiadis, B.J. Marinas, and A.M. Mayes, Nature 452, 301 (2008).

[8] K. Buruga, J.T. Kalathi, K. Kim, Y.S. Ok, and B. Danil, J Ind Eng Chem 61, 169 (2018).

[9] Z. Guo, T.S. Zhao, C. Xu, and Y. Shi, Int J Comput Fluid D 20, 361, 1 (2006).

[10] L. Chen, J. Wen, P. Zhang, B. Yu, C. Chen, T. Ma, X. Lu, S.H. Kim, and L. Qian, Nat Commun 9 (2018).

[11] J.D. Figueroa, T. Fout, S. Plasynski, H. McIlvried, and R.D. Srivastava, Int J Greenh Gas Con 2, 9 (2008).

[12] M. Konarova, A. Tanksale, J. Norberto Beltramini, and G. Qing Lu, Nano Energy 2, 98 (2013).

[13] B. Li, H.M. Wen, Y. Yu, Y. Cui, W. Zhou, B. Chen, and G. Qian, Materials Today Nano 2, 21 (2018).

[14] Y. Zhang, L. Zhu, R. Wang, and Z. Guo, Phys Rev E 97, 53306 (2018).

[15] K. Xu and J. Huang, J Comput Phys 229, 7747 (2010).

[16] Z. Guo, J. Qin, and C. Zheng, Phys Rev E 89, 13021, 1 (2014).

[17] S. Shen, G. Chen, R.M. Crone, and M. Anaya-Dufresne, Phys Fluids 19, 86101 (2007).

[18] W. Zhang, G. Meng, and X. Wei, Microfluid Nanofluid 13, 845 (2012).

[19] K.P. Travis and K.E. Gubbins, J Chem Phys 112, 1984 (2000). 
[20] S. Roy, R. Raju, H.F. Chuang, B.A. Cruden, and M. Meyyappan, J Appl Phys 93, 4870, 1 (2003).

[21] M. Kazemi and A. Takbiri-Borujeni, Int J Coal Geol 146, 188 (2015).

[22] Z. Guo, T.S. Zhao, and Y. Shi, Phys Rev E 71, 35301, 1 (2005).

[23] S. Chapman and T.G. Cowling, The mathematical theory of non-uniform gases (Cambridge University Press, 1970).

[24] H. Van Beijeren and M.H. ERNST, Physica 68, 437 (1973).

[25] J. Karkheck and G. Stell, J Chem Phys 75, 1475 (1981).

[26] M. Grmela, J Stat Phys 3, 347 (1971).

[27] M. Sadr and M.H. Gorji, J Comput Phys 378, 129 (2019).

[28] H.T. Davis, J Chem Phys 86, 1474 (1987).

[29] T.K. Vanderlick, L.E. Scriven, and H.T. Davis, J Chem Phys 90, 2422 (1989).

[30] T.K. Vanderlick and H.T. Davis, J Chem Phys 87, 1791 (1987).

[31] Z. Guo, K. Xu, and R. Wang, Phys Rev E 88, 33305 (2013).

[32] G.R. McNamara and G. Zanetti, Phys Rev Lett 61, 2332 (1988).

[33] Z. Guo, R. Wang, and K. Xu, Phys Rev E 91, 33313, 1 (2015).

[34] P. Wang, S. Tao, and Z. Guo, Comput Fluids 120, 70 (2015).

[35] Z. Guo and K. Xu, Int J Heat Mass Tran 102, 944 (2016).

[36] X. Luo and H. Yi, Int J Heat Mass Tran 114, 970 (2017).

[37] C. Zhang and Z. Guo, Int J Heat Mass Tran 134, 1127 (2019).

[38] L. Zhu and Z. Guo, Comput Fluids (2017).

[39] H. Liu, Y. Cao, Q. Chen, M. Kong, and L. Zheng, Comput Fluids 167, 313 (2018).

[40] Y. Huo and Z. Rao, Int Commun Heat Mass 91, 187 (2018). 
[41] C. Zhang, K. Yang, and Z. Guo, Int J Heat Mass Tran 126, 1326 (2018).

[42] Z. GUO, J. LI, and K. XU, arXiv preprint arXiv:1909.04923 (2019).

[43] J.W. Dufty, A. Santos, and J.J. Brey, Phys Rev Lett 77, 1270 (1996).

[44] Z. Guo, T.S. Zhao, and Y. Shi, Phys Fluids 18, 67107 (2006).

[45] P. Tarazona, Phys Rev a 31, 2672 (1985).

[46] N.F. Carnahan and K.E. Starling, J Chem Phys 51, 635 (1969).

[47] W.A. Steele, Surf Sci 36, 317 (1973).

[48] X. He, X. Shan, and G.D. Doolen, Phys Rev E 57, R13, 1 (1998).

[49] J.A. Barker and D. Henderson, J Chem Phys 47, 4714 (1967).

[50] R.L. Cotterman, B.J. Schwarz, and J.M. Prausnitz, AICHhE J, 1787 (1986).

[51] G. Strang, Siam J Numer Anal 5, 506 (1968).

[52] S. Tao, H. Zhang, Z. Guo, and L. Wang, J Comput Phys 375, 498 (2018).

[53] P. Wang, M.T. Ho, L. Wu, Z. Guo, and Y. Zhang, Comput Fluids 161, 33 (2018).

[54] B. Van Leer, J Comput Phys 23, 276 (1977).

[55] I. Bitsanis, J.J. Magda, M. Tirrell, and H.T. Davis, J Chem Phys 87, 1733 (1987).

[56] K. Wu, Z. Chen, J. Li, X. Li, J. Xu, and X. Dong, P Natl Acad Sci Usa 114, 3358 (2017).

[57] I. Bitsanis, S.A. Somers, H.T. Davis, and M. Tirrell, J Chem Phys 93, 3427 (1990).

[58] I.K. Snook and W. van Megen, J Chem Phys 72, 2907 (1980). 